\title{
ON NECESSARY AND SUFFICIENT CONDITIONS FOR VARIABLE EXPONENT HARDY INEQUALITY
}

\author{
AZIZ HARMAN
}

Abstract. In this paper we derive close necessary and sufficient conditions on the regularity of the exponent functions $p, \beta$ such that the variable exponent Hardy inequality holds

$$
\left\|x^{\beta(x)-1} \int_{0}^{x} f(t) d t\right\|_{L^{p(\cdot)}(0, l)} \leqslant C\left\|x^{\beta(x)} f\right\|_{L^{p(.)}(0, l)}
$$

Mathematics subject classification (2010): 42A05, 42B25, 26D10, 35A23.

Keywords and phrases: Hardy operator, Hardy inequality, variable exponent, weighted inequality.

\section{REFERENCES}

[1] D. CRUZ.-URIBe, SFO AND F. I. MAmedov, On a general weighted Hardy type inequality in the variable exponent Lebesgue spaces, Revista Mat. Comp., 25(2) (2012), 335-367.

[2] L. Diening, P. Harjulehto, P. Hasto, M. RuZicka, Lebesgue and Sobolev Spaces with Variable Exponents, Lecture Notes in Mathematics, vol. 2017. Springer, Berlin (2011).

[3] L. Diening And S. SAmKo, Hardy inequality in variable exponent Lebesgue spaces, Fract. Calc. and Appl. Anal., 10(1) (2007), 1-17.

[4] D. E. Edmunds, V. Kokilashvili And A. Meskhi, On the boundedness and compactness of the weighted Hardy operators in spaces $L^{p(x)}$, Georgian Math. J., 12(1) (2005), 27-44.

[5] X. L. FAn AND D. ZhaO, On the spaces $L^{p(x)}(\Omega)$ and $W^{m, p(x)}(\Omega)$, J. Math. Anal. Appl., 263 (2001), $424-446$.

[6] P. Harjulehto, P. Hasto And M. Kos Kinoja, Hardy's inequality in variable exponent Sobolev spaces, Georgian Math. J., 12(3) (2005), 431-442.

[7] A. Harman AND, F. I. Mamedov, On boundedness of weighted Hardy operator in $L^{p(.)}$ and regularity condition, J. Ineq. Appl., vol 2010, 14 pages.

[8] O. KovaciK AND J. RAKosniK, On spaces $L^{p(x)}$ and $W^{p(x)}$, Czech. Math. J., 41(116) (1991), 592 618.

[9] A. Kufner And L. E. Persson, Weighted inequalities of Hardy type, World Scientific, New JerseyLondon-Singapore-Hong Kong (2003).

[10] F. I. Mamedov And A. HaRman, On a weighted inequality of Hardy type in spaces $L^{p(.)}$, J. Math. Anal. Appl., 353(2) (2009), 521-530.

[11] F. I. Mamedov And A. Harman, On a Hardy type general weighted inequality in spaces $L^{p(.)}$, Int. Eq. Oper. Theor., 66(4) (2010), 565-592.

[12] F. I. Mamedov And Y. Zeren, On equivalent conditions for the general weighted Hardy type inequality in space $L^{p(.)}$, Z. Anal. Anwen., 31(1) (2012), 55-74.

[13] F. I. MAMEDOv, On Hardy type inequality in variable exponent Lebesgue space $L^{p(.)}(0,1)$, Azerb. J. of Math. 2(1) (2012), 90-99.

[14] R. Mashiyev, B. Cekic, F. I. Mamedov and S. Ogras, Hardy's inequality in power-type weighted $L^{p(.)}$ spaces, J. Math. Anal. Appl., 334(1) (2007), 289-298.

[15] H. Rafeiro, S. G. Samko, Hardy inequality in variable Lebesgue spaces, Ann. Acad. Sci. Fen.Math., 34 (1) (2009), 279-289. 
[16] S. G. SAmко, Hardy inequality in the generalized Lebesgue spaces, Fract. Calc. Appl. Anal., 6 (4) (2003), 355-362.

[17] S. G. S AMKo, Convolution type operators in $L^{p(.)}$, Int. Tran. Spec. Fun., 7 (1998), 123-144. 